\title{
Gauge group and reality conditions in Ashtekar's complex formulation of canonical gravity
}

\author{
J. M. Pons* \\ Departament d'Estructura i Constituents de la Matèria, Universitat de Barcelona, and Institut de Física d'Altes Energies, \\ Diagonal 647, E-08028 Barcelona, Catalonia, Spain \\ D. C. Salisbury ${ }^{\dagger}$ \\ Department of Physics, Austin College, Sherman, Texas 75090-4440 \\ L. C. Shepley \\ Center for Relativity, Physics Department, The University of Texas, Austin, Texas 78712-1081
}

(Received 2 August 1999; published 25 August 2000)

\begin{abstract}
We discuss reality conditions and the relation between spacetime diffeomorphisms and gauge transformations in Ashtekar's complex formulation of general relativity. We produce a general theoretical framework for the stabilization algorithm for the reality conditions, which is different from Dirac's method of stabilization of constraints. We solve the problem of the projectability of the diffeomorphism transformations from configuration-velocity space to phase space, linking them to the reality conditions. We construct the complete set of canonical generators of the gauge group in the phase space which includes all the gauge variables. This result proves that the canonical formalism has all the gauge structure of the Lagrangian theory, including the time diffeomorphisms.
\end{abstract}

PACS number(s): 04.20.Fy, 11.10.Ef

\section{INTRODUCTION}

In recent papers [1-3] we have discussed some special features exhibited by the gauge groups in Einstein and Einstein-Yang-Mills theories and in a real triad approach to general relativity when their formulations are brought from configuration-velocity space (the tangent bundle $T Q$ ) to phase-space (the cotangent bundle $T^{*} Q$ ). Our viewpoint is that the configuration-velocity space and phase space formulations are equivalent (see [4]). We found that some of the generators of the diffeomorphism group in the tangent bundle are not projectable to the cotangent bundle. To make them projectable, the otherwise arbitrary functions in the gauge group generators must depend on the field variables, particularly on the lapse function and shift vector of the metric - though this dependence still allows all infinitesimal diffeomorphisms to be represented. In Einstein-Yang-Mills and triad theories, diffeomorphisms must be accompanied by other gauge transformations in order to be projectable. When projectability is achieved, we have the full proof that indeed the gauge group is the same in configuration-velocity space as in phase space; this identity of the gauge group is not widely recognized.

Here we study in detail the issue of the gauge group in the Ashtekar complex formulation [5-7] of canonical gravity. Ashtekar's use of a self-dual connection makes this formulation very similar to a Yang-Mills theory, and so we expect to get and do get results similar to our previous results. However, a somewhat unusual aspect of this program is the use of a complex Lagrangian and a complex Hamiltonian. The fact that Ashtekar's connection is complex introduces essential

\footnotetext{
*Electronic address: pons@ecm.ub.es

†Electronic address: dsalisbury@austinc.edu

‡Electronic address: larry@einstein.ph.utexas.edu
}

novelties. To recover real gravity, reality conditions must be imposed, and we make a thorough examination of them. These conditions are not constraints in a Dirac sense $[8,9]$. We develop the theoretical framework for a stabilization algorithm to maintain the reality conditions under time evolution. This algorithm is different from the Dirac stabilization algorithm for constraints because of the complex character of the Hamiltonian, though our treatment is conceptually close to Dirac's method.

Recently generalizations of Ashtekar's complex formalism have been introduced. In one approach it has been shown that general relativity can be reformulated as a one-parameter family of real connections [10-12]. When the otherwise real parameter takes the value $i$, one recovers the Ashtekar complex connection. However, one apparent drawback to this real approach is that the scalar constraint loses the simple form it assumes in the complex regime. This could constitute a serious obstacle for the quantization program, though it is true that difficulties in constructing a Hilbert space satisfying the reality conditions in the complex Ashtekar program are thereby circumvented. A second approach undertakes a generalized Wick transform of the complex connection to a real connection [13,14]. This transform has been shown under certain circumstances to be equivalent to an analytic continuation to imaginary time [15], and thus to a spacetime with Riemannian signature. The advantage one hopes to gain through this transform is that it may be possible to solve the simpler scalar constraint in the Lorentzian sector and then implement the Wick transform, thus satisfying the reality conditions.

The argument we put forth here is that the relevance of the complex Ashtekar approach has certainly not diminished. A major theme in this paper is the relation of the scalar constraint to spacetime diffeomorphisms.

Our purposes in this paper are twofold: On the one hand, we will clarify the structure of the generators of the gauge 
group in the complex Ashtekar formulation of canonical gravity. On the other hand, we will discuss fully the stabilization algorithm for the reality conditions. It is not surprising - perhaps - that both aspects, gauge group and reality conditions, are related: Any symmetry, including gauge symmetries must preserve the reality conditions. We will exhibit the links that exist between these conditions and the conditions of projectability from configuration-velocity to phase space of gauge variations. We distinguish between metric reality conditions (only the full spacetime metric itself must be real) and triad reality conditions (the spatial orthonormal triad vectors, as well as the metric, must be real) as in $[16,17]$. We will see that the rotation gauge group (for the triads) is reduced from $\mathrm{SO}(3, C)$ to $\mathrm{SO}(3, R)$ to fulfill the triad reality conditions. Our results concerning the reality conditions do agree with those of [17]; our contribution is that we make clear when the stabilization algorithm for the reality conditions is terminated and how it applies in a general sense. Also, we give a thorough discussion of the elimination of part of the gauge freedom when we extend reality conditions from metric to triad.

We explicitly assume that the connection $A_{\mu}^{i}$ is complex but also consider the possibility that all variables in phase space are complex. It is significant that all the gauge variables, that is the lapse, the shift, and the time component of the connection $A_{0}^{i}$, are retained as canonical variables in the analysis of gauge symmetries which we will present. In particular, it could well prove useful in quantum gravity to retain $A_{0}^{i}$ as an operator. We would thus contemplate holonomies, parallel transporters of $\mathrm{SU}(2)$, in directions off the constant-time hypersurfaces. We presume that all functions, including the Hamiltonian, are analytic, and that phase space has a standard Poisson bracket structure. Physical reasons require that some of the variables must be real. Then it is necessary to impose restrictions on the initial conditions and to restrict gauge freedom in such a way that time evolution will keep real these variables. These restrictions are called the reality conditions.

This paper is organized as follows: The stabilization algorithm for the reality conditions is presented in Sec. II. The algorithm is general in the sense that it can be applied to any complex theory in which physical reasons require that some of the variables be real. In Sec. III, the Ashtekar approach is succinctly introduced with some results and notations. The canonical approach is undertaken in Sec. IV, and in Sec. V we apply the reality condition algorithm to the case of Ashtekar canonical gravity. In Secs. VI and VII we solve the problem of finding the projectable gauge transformations and their canonical generators, finding in the process some interesting relations with the reality conditions. We discuss the counting of degrees of freedom in Sec. VIII. We devote Sec. IX to conclusions.

\section{STABILIZATION ALGORITHM FOR REALITY CONDITIONS-GENERAL THEORY}

In this section we provide the theoretical setting for what properly must be called the stabilization algorithm for the reality conditions. This setting is applicable to any dynamical theory that makes use of complex variables but requires that some of these variables be real to be physically acceptable. In other words, initial conditions must fix real values for these variables, and time evolution must preserve the reality.

Reality conditions are not constraints in the Dirac sense. The difference comes from the fact that reality conditions do not place restrictions on the variables of the formalism but only on the values of some real or imaginary parts of these variables. The difference is made even more clear when we consider stabilization procedures. If the Dirac Hamiltonian is, say, $H$, the stabilization of a (time independent) Diractype constraint $\phi$ is to require the tangency of the dynamical vector field $\{-, H\}$ on the surface defined by $\phi=0$ :

$$
\{\phi, H\}=0 .
$$

This requirement may introduce new constraints or the determination some arbitrary functions in $H$. The stabilization of a Dirac constraint follows this procedure whether $H$ is real or complex.

Instead, if we have a (time independent) reality condition, such as the vanishing of the imaginary part of a quantity $f$, $\mathfrak{F} f=0$, its stabilization involves, at least, the requirement

$$
\mathfrak{F}\{f, H\}=0 .
$$

This is not a tangency condition. Moreover, the expression

$$
\{\mathfrak{F} f, H\}
$$

makes no sense at all in the formalism, because the bracket is defined for complex phase space variables and cannot be applied to real or imaginary parts of these variables.

Before developing the correct stabilization for reality conditions, we briefly review the basics of the stabilization algorithm for Dirac constraints. Similarities and differences between the two stabilization procedures will become evident.

\section{A. Stabilization of Dirac constraints}

Dirac's method applies both to the Lagrangian and Hamiltonian formalisms, but here we will only consider its implementation in the latter case. Consider a dynamical evolution in phase space with some gauge freedom. We start with the canonical Hamiltonian $H_{\mathrm{c}}$, whose pullback to configurationvelocity space is the Lagrangian energy

$$
E_{L}:=\dot{q}^{i} \frac{\partial L}{\partial \dot{q}^{i}}-L,
$$

where $L$ is the Lagrangian, which we take to be timeindependent, $\left\{q^{i}\right\}$ are the configuration components, and is $d / d t$. The Dirac Hamiltonian is

$$
H_{\mathrm{D}}=H_{\mathrm{c}}+\lambda^{\mu} \phi_{\mu} ;
$$

the $\phi_{\mu}$ are the primary constraints, $\mu=1, \ldots, n$, and $\lambda^{\mu}$ are Lagrange multipliers (arbitrary functions in principle) that describe the gauge freedom available to this system. The first step in Dirac's method is to ask for the dynamics to result in 
trajectories tangent to the primary constraint surface. This requirement of tangency may lead to the determination of some of the multipliers $\lambda^{\mu}$ and the appearance of new constraints. The next step is again to require that the trajectories be tangent to the new constraint surface. The stabilization procedure continues and eventually is completed.

We analyze this procedure from the point of view of finite time evolution for application in Sec. II C. To make things simpler, as an example, we assume that none of the multipliers $\lambda^{\mu}$ are determined at any step of the above procedure. Then, as far as the time-evolution of the constraints is concerned, we can use the time-independent $H_{\mathrm{c}}$ as the dynamical generator. We start with the primary constraints $\phi_{\mu}$. The time evolution operator from time zero to time $t$ is

$$
E[t]=\exp \left(t\left\{-, H_{\mathrm{c}}\right\}\right),
$$

with the expansion

$$
\begin{aligned}
\phi_{\mu}[t]= & E[t] \phi_{\mu} \\
= & \phi_{\mu}+t\left\{\phi_{\mu}, H_{\mathrm{c}}\right\}+\frac{t^{2}}{2}\left\{\left\{\phi_{\mu}, H_{\mathrm{c}}\right\}, H_{\mathrm{c}}\right\} \\
& +\frac{t^{3}}{3 !}\left\{\left\{\left\{\phi_{\mu}, H_{\mathrm{c}}\right\}, H_{\mathrm{c}}\right\}, H_{\mathrm{c}}\right\}+\ldots \\
= & \sum_{n=0}^{\infty} \frac{t^{n}}{n !}\left\{\phi_{\mu}, H_{\mathrm{c}}\right\}_{(n)} ;
\end{aligned}
$$

in this expression $\phi_{\mu}[t]$ is the function $\phi_{\mu}(x(t))$, where $x(t):=(q(t), p(t))$ is the trajectory in phase space satisfying the equations

$$
\dot{x}(t)=\left.\left\{x, H_{\mathrm{c}}\right\}\right|_{x=x(t)} .
$$

To preserve the primary constraints under finite evolution we must require

$$
\phi_{\mu}[t]=0
$$

for any $t$. This is the same as the infinite set of restrictions

$$
\left\{\phi_{\mu}, H_{\mathrm{c}}\right\}_{(n)}=0 ;
$$

note that $n=0$ corresponds to the primary constraints $\phi_{\mu}$ $=0$.

In general, the $n=1$ level of stabilization in Eq. (2.4), $\left\{\phi_{\mu}, H_{\mathrm{c}}\right\}=0$, may introduce new independent constraints (secondary constraints) $\phi_{\mu}^{(1)}:=\left\{\phi_{\mu}, H_{\mathrm{c}}\right\}$. The second level of stabilization is $\left\{\phi_{\mu}^{(1)}, H_{\mathrm{c}}\right\}=0$, which is Dirac's requirement that the vector field $\left\{-, H_{\mathrm{c}}\right\}$ be tangent to the new constraint surface (defined by all the primary and secondary constraints). It is worth noticing that in general the algorithm to get new constraints will eventually stop, and only a finite number of the requirements in Eq. (2.4) will be relevant.

For instance, if there are no tertiary constraints, the $n$ $=2$ level of stabilization is satisfied when the primary and secondary constraints are taken into account. Then, $\left\{\phi_{\mu}^{(1)}, H_{\mathrm{c}}\right\}$ is a linear combination of the primary and secondary constraints. All other terms in Eq. (2.4) vanish under the condition that all of the primary and secondary constraints are satisfied. There are exceptions to this casual statement, in particular when some of the constraints are not effective (an effective constraint has nonvanishing differential on the constraint surface), and we discuss them in the next section. With these exceptions, the stabilization procedure terminates when we find a level of stabilization that is already satisfied under the requirements introduced in the previous levels.

The general situation is when we must consider time dependence in $H_{D}$ (because of the $\left.\lambda^{\mu}\right)$. In this case, $H_{\mathrm{D}}\left(t_{1}\right)$ does not necessarily have vanishing Poisson bracket with $H_{\mathrm{D}}\left(t_{2}\right)$, for $t_{1} \neq t_{2}$. The time evolution operator (2.2) is then replaced by

$$
E[t]=\mathcal{T} \exp \left(\int_{0}^{t} d t^{\prime}\left\{-, H_{\mathrm{D}}\left(t^{\prime}\right)\right\}\right),
$$

where $\mathcal{T}$ is the time-ordering operator: It acts as

$$
\mathcal{T}\left\{\left\{-, H_{\mathrm{D}}\left(t_{1}\right)\right\}, H_{\mathrm{D}}\left(t_{2}\right)\right\}=\left\{\left\{-, H_{\mathrm{D}}\left(t_{<}\right)\right\}, H_{\mathrm{D}}\left(t_{>}\right)\right\},
$$

with $t_{>}=\max \left(t_{1}, t_{2}\right)$ and $t_{<}=\min \left(t_{1}, t_{2}\right)$ (this expression generalizes to any order).

The levels of stabilization in Eq. (2.5) now become

$$
\begin{array}{r}
\left\{\phi_{\mu}, H_{\mathrm{D}}(t)\right\}=0, \\
\left\{\left\{\phi_{\mu}, H_{\mathrm{D}}\left(t_{1}\right)\right\}, H_{\mathrm{D}}\left(t_{2}\right)\right\}=0, \\
\left\{\left\{\left\{\phi_{\mu}, H_{\mathrm{D}}\left(t_{1}\right)\right\}, H_{\mathrm{D}}\left(t_{2}\right)\right\}, H_{\mathrm{D}}\left(t_{3}\right)\right\}=0, \\
\ldots,
\end{array}
$$

with $t_{1}<t_{2}<t_{3}<\ldots$. These requirements (2.6) may determine some of the arbitrary functions in $H_{\mathrm{D}}$ or they may bring forth further constraints. Once an arbitrary function gets determined, it can be replaced by its expression in phase space for all remaining levels of stabilization.

The sequence (2.6) eventually terminates when the stabilization equations for all the constraints no longer determine new constraints: Higher stabilization equations are automatically satisfied.

\section{B. An aside on ineffective constraints}

There is an exception to the rule, just enunciated, that says that the stabilization algorithm is finished when, at a given level, no new constraints appear. The expression $\left\{\phi_{\mu}^{(1)}, H\right\}$ $=0$ is meant to be Dirac's requirement that the vector field $\{-, H\}$ be tangent to the constraint surface defined by the primary and secondary constraints. This is not an accurate statement when a secondary constraint is ineffective (the primary constraints are always taken in effective form), that is, if its differential vanishes on the constraint surface. For instance, consider the effective constraint $\phi$. To make it ineffective we can square it to get $f=\phi^{2}$. The two constraints still define the same surface, $\phi=0 \Leftrightarrow f=0$. However, the vanishing of $\{f, H\}$ does not imply the tangency of $\{-, H\}$ to the surface $f=0$ but rather a triviality, because $\{f, H\}$ $=2 \phi\{\phi, H\}$ automatically vanishes on $f=0$. This reflects the ineffective character of $f$ (but notice that $\{f, H\}$ cannot be 
expressed as a linear combination of $f$ with the coefficient being regular at the surface $f=0$ ).

Because of the possible presence of ineffective constraints, it may be true that one level of stabilization does not bring new restrictions, and yet subsequent levels do. In fact, in our example with $f$ ineffective, the next level of stabilization produces $\{\{f, H\}, H\}=2 \phi\{\{\phi, H\}, H\}+2\{\phi, H\}^{2}$. This could introduce a new ineffective constraint $\{\phi, H\}^{2}=0$ that defines the same surface as $\{\phi, H\}=0$.

The moral is that if we have ineffective constraints, we must take special precautions that the tangency conditions are correctly implemented and that all levels of Eq. (2.6) are examined.

\section{Stabilization of reality conditions}

Suppose that our reality condition requires that the functions $f_{\alpha}$, for some set of indices $\alpha$, must be kept real under time evolution. We begin, for simplicity, with the case when the Lagrangian multipliers play no part, as in Sec. II A; then we may work with the time-independent canonical Hamiltonian $H_{\mathrm{c}}$. Expressed in the notation introduced above, the reality requirement is

$$
\mathfrak{F}\left(f_{\alpha}[t]\right)=0,
$$

which is, using the evolution operator (2.3),

$$
\mathfrak{F}\left(f_{\alpha}[t]\right)=\mathfrak{F}\left(E[t] f_{\alpha}\right)=\sum_{n=0}^{\infty} \frac{t^{n}}{n !} \mathfrak{F}\left\{f_{\alpha}, H_{\mathrm{c}}\right\}_{(n)}=0,
$$

for any $t$. Therefore, in addition to the primary reality condition,

$$
\mathfrak{F} f_{\alpha}=0,
$$

we get the levels of stabilization

$$
\begin{array}{r}
\mathfrak{F}\left\{f_{\alpha}, H_{\mathrm{c}}\right\}=0, \\
\mathfrak{F}\left\{\left\{f_{\alpha}, H_{\mathrm{c}}\right\}, H_{\mathrm{c}}\right\}=0, \\
\mathfrak{F}\left\{\left\{\left\{f_{\alpha}, H_{\mathrm{c}}\right\}, H_{\mathrm{c}}\right\}, H_{\mathrm{c}}\right\}=0, \\
\ldots
\end{array}
$$

We call these conditions the secondary reality condition, tertiary reality condition, and so on. Notice in fact that all these requirements need only to hold on the constraint surface, because the complete dynamical setting is given by the evolution operator (2.2) supplemented with the Dirac constraints.

One striking difference between these conditions (2.8) and the Dirac stability conditions (2.6) is that the vanishing of one level of stabilization due to the fulfillment of the previous ones does not guarantee that the subsequent levels will also vanish. For instance, let us suppose that

$$
\mathfrak{F}\left\{f_{\alpha}, H_{\mathrm{c}}\right\}=\eta_{\alpha}^{\beta} \mathfrak{F}\left(f_{\beta}\right),
$$

for a real matrix $\eta_{\alpha}^{\beta}$ (in field theory, the summation over like indices implies a spatial integration, also), so that the secondary reality condition is satisfied when the primary one is. However, this relation is of no value in implementing the tertiary condition. Instead, if we had

$$
\left\{f_{\alpha}, H_{\mathrm{c}}\right\}=\eta_{\alpha}^{\beta} f_{\beta}
$$

for any real matrix $\eta_{\alpha}^{\beta}$ such that

$$
\left\{\eta_{\alpha}^{\beta}, H_{\mathrm{c}}\right\}=0,
$$

then indeed the stabilization algorithm would have been over. Of course this is only a sufficient condition.

In a more realistic case we would use $H_{\mathrm{D}}$, which is in general time dependent. Considering how we arrived at Eq. (2.8), which plays, for the reality conditions, the role analogous to Eq. (2.4) for Dirac constraints, it is easy to get an analog for Eq. (2.6). In fact we can use here all the results obtained from the Dirac analysis, in particular the determination in phase space of some of the Lagrange multipliers. This means that we can start with a first class $(f c)$ Hamiltonian

$$
H_{\mathrm{D}}^{f c}=H_{\mathrm{c}}+\sum_{\mu=1}^{n_{1}} \lambda_{\mathrm{c}}^{\mu} \boldsymbol{\phi}_{\mu}+\sum_{\mu=n_{1}}^{n} \lambda^{\mu} \boldsymbol{\phi}_{\mu}
$$

where we have assumed for simplicity that the first $n_{1}$ Lagrange multipliers are the ones that get determined as functions $\lambda_{c}^{\mu}$ in phase space through the Dirac stabilization algorithm. In this general case the reality conditions may lead to a further reduction of the gauge freedom present in $H_{\mathrm{D}}^{f c}$, that is, to a partial determination of the remaining Lagrange multipliers-for instance: their real or imaginary parts. This is what will happen with the triad reality conditions for the Ashtekar formulation, to be analyzed in Sec. V.

It is obvious that nothing in this section depends on the theory being formulated in phase space. Indeed, we could replace $\left\{-, H_{\mathrm{c}}\right\}+\lambda^{\mu}\left\{-, \phi_{\mu}\right\}$ everywhere by $\mathbf{X}+\lambda^{\mu} \mathbf{Y}_{\mu}$, with $\mathbf{X}$ and $\mathbf{Y}_{\mu}$ being vector fields in some given space (for instance configuration-velocity space).

\section{THE ASHTEKAR LAGRANGIAN}

One way to present the Ashtekar Lagrangian density is [18-21]

$$
\mathcal{L}_{A}={ }^{4} F_{\mu \nu}^{I J}\left[{ }^{4} A\right] E_{I}^{\mu} E_{J}^{\nu} \sqrt{|g|}
$$

where $g$ is the determinant of the spacetime metric; $E_{I}^{\mu}$ are the tetrad components, $\mu$ being a spacetime index and $I$ an internal index; and ${ }^{4} F_{\mu \nu}^{I J}$ is the curvature tensor associated with the Ashtekar connection ${ }^{4} A_{\mu}^{I J}$. We use the standard definitions of these quantities [22], and we do not repeat these definitions here, because we will be working in a 3 +1 decomposition and will give specific definitions of our variables below.

$\mathcal{L}_{A}$ is interpreted in a Palatini-like formalism: The components of the self-dual complex connection are taken to be 
independent variables. Their equations of motion determine them in terms of the other variables (and their derivatives). This determination is similar to the determination of the Christoffel coefficents in the Einstein-Palatini version of general relativity (see [23] for a good review of actions for gravity). Variables having this property of being determined by their own equations of motion are usually called auxiliary variables. When this dynamical determination of the Ashtekar connection is substituted into the Lagrangian we get the standard Ashtekar Lagrangian, which is equivalent to the Einstein-Hilbert Lagrangian.

We are interested in the canonical description (in phase space). Therefore we will write the action in a $3+1$ decomposition of the variables. The contravariant spacetime metric is written in terms of the lapse function $N$ and shift vector $N^{a}$, and a triad of orthonormal vectors $T_{i}^{a} \quad(a, b$ are spatial indices; $i, j$ are internal indices, raised or lowered with $\delta_{i j}$, so that repeated internal indices imply a sum even if both are raised or lowered):

$$
g^{\mu \nu}=\left(\begin{array}{cc}
-N^{-2} & N^{-2} N^{a} \\
N^{-2} N^{b} & T_{i}^{a} T_{i}^{b}-N^{-2} N^{a} N^{b}
\end{array}\right) .
$$

The triad vectors and the (unit) normal vector to the constant-time hypersurfaces

$$
n^{\mu}=\left(N^{-1},-N^{-1} N^{a}\right)
$$

constitute an orthonormal tetrad.

We represent the components of the orthonormal spatial one-forms by $t_{a}^{i}$, so that the covariant three-metric is given by

$$
g_{a b}=t_{a}^{i} t_{b}^{i}
$$

It turns out to be convenient to take one set of canonical variables to be the triad vectors multiplied by the square root of the determinant of the three-metric. As has now become conventional, we represent densities of arbitrary positive weight under spatial diffeomorphisms by an appropriate number of tildes over the symbol. For negative weights we place the tilde(s) below the symbol. Hence we define, for

$$
t:=\sqrt{\operatorname{det}\left(g_{a b}\right)}=\operatorname{det}\left(t_{a}^{i}\right),
$$

the densitized triad as

$$
\widetilde{T}_{i}^{a}:=t T_{i}^{a}
$$

In the Ashtekar approach the connection is self-dual. An antisymmetric tensor, whose components in an orthonormal tetrad are $F_{I J}$, is self-dual if

$$
i F_{I J}=\frac{1}{2} \epsilon_{I J K L} F^{K L},
$$

where $\epsilon_{I J K L}$ is the four-dimensional Levi-Civita symbol defined by $\epsilon_{0123}=-1$. Because of self-duality, the fourconnection ${ }^{4} A_{\mu}^{I J}$ in Eq. (3.1) is determined by the independent components

$$
A_{\mu}^{i}:=\frac{1}{2} \epsilon^{i j k 4} A_{\mu}^{j k},
$$

$\epsilon^{i j k}$ being the Levi-Civita symbol. In the $3+1$ decomposition the Ashtekar Lagrangian becomes $\left({ }^{\circ}\right.$ is $\partial / \partial x^{0}$, and we will also use a subscript comma for partial derivatives)

$$
\mathcal{L}_{A}=i \dot{\widetilde{T}}_{i}^{a} A_{a}^{i}-i A_{0}^{i} \mathcal{D}{ }_{a} \widetilde{T}_{i}^{a}+i N^{a} \widetilde{T}_{i}^{b} F_{a b}^{i}+\frac{1}{2} N \widetilde{\sim}_{i}^{a} \widetilde{T}_{j}^{b} F_{a b}^{i j}
$$

where $F_{a b}^{j k}=: \epsilon^{i j k} F_{a b}^{i}$ is the three dimensional Riemann tensor associated with the Ashtekar connection,

$$
F_{a b}^{i}:=A_{b, a}^{i}-A_{a, b}^{i}-\epsilon^{i j k} A_{a}^{j} A_{b}^{k}
$$

and where the covariant derivative $\mathcal{D}_{b}$ is defined using the Ashtekar connection: Its action on the densitized triad is, for example,

$$
\mathcal{D}_{b} \widetilde{T}_{i}^{a}={ }^{3} \nabla_{b} \widetilde{T}_{i}^{a}+\epsilon^{i j k} \widetilde{T}_{j}^{a} A_{b}^{k},
$$

${ }^{3} \nabla_{b}$ being the covariant derivative based on the 3 -metric $g_{a b}$. It is convenient to take the densitized lapse $\underset{\sim}{N}$ as an independent variable, but for convenience, some equations will be written in terms of $N$ itself; likewise it will prove convenient to use both densitized and undensitized variables in some of our results.

Two observations should be made at this point.

First: From the fact that $\mathcal{L}$ in Eq. (3.4) does not depend on the velocities $\underset{\sim}{\dot{N}}, \dot{N}^{a}, \dot{A}_{0}^{i}$, we can conclude (details are given in [1]) that the necessary and sufficient condition for a function $f$ in configuration-velocity space $T Q$ to be projectable to phase space $T^{*} Q$ is that $f$ does not depend on these velocities.

Second: The fact that the independent components of the Ashtekar connection play the role of auxiliary variables tells us that their equations of motion give

$$
A_{\mu}^{i}-\Omega_{\mu}^{i}-i \Omega_{\mu}^{0 i}=0
$$

where $\Omega_{\mu}^{i}:=\frac{1}{2} \epsilon^{i j k} \Omega_{\mu}^{j k}$ and $\Omega_{\mu}^{0 i}$ are the components of the spin connection, that is, the Ricci rotation coefficients. In particular, $\Omega_{a}^{i j}$ are the three-dimensional Ricci rotation coefficients formed from the triad, so that

$$
\Omega_{a}^{i}:=\frac{1}{2} \epsilon^{i j k} t_{b}^{j}\left(T_{k, a}^{b}+{ }^{3} \Gamma_{c a}^{b} T_{k}^{c}\right)=: \omega_{a}^{i}
$$

with ${ }^{3} \Gamma_{c a}^{b}$ being the Christoffel symbols. For future use, we define the covariant derivative using the three-dimensional Ricci coefficients, which applied to $\widetilde{T}_{i}^{a}$ gives zero:

$$
D_{b} \widetilde{T}_{i}^{a}={ }^{3} \nabla_{b} \widetilde{T}_{i}^{a}+\epsilon^{i j k} \widetilde{T}_{j}^{a} \omega_{b}^{k}=0 .
$$

Notice that when Eq. (3.6) holds,

$$
\left(\mathcal{D}_{a}-D_{a}\right) \widetilde{T}_{i}^{b}=i \epsilon^{i j k} \widetilde{T}_{j}^{b} \Omega_{a}^{0 k}
$$


The other components of the spin connection involve time derivatives:

$$
\begin{aligned}
& \Omega_{0}^{i}:=\frac{1}{2} \epsilon^{i j k}\left(\dot{t}_{a}^{j} T_{k}^{a}+N_{, a}^{b} t_{b}^{k} T_{j}^{a}+t_{b, a}^{k} T_{j}^{a} N^{b}+t_{b, a}^{l} N^{c} t_{c}^{l} T_{j}^{a} T_{k}^{b}\right), \\
& \Omega_{a}^{0 i}:=T_{i}^{b} K_{a b}, \\
& \Omega_{0}^{0 i}:=T_{i}^{a} N_{, a}+N^{a} T_{i}^{b} K_{a b},
\end{aligned}
$$

where $K_{a b}$ is the extrinsic curvature, defined as

$$
K_{a b}:=\frac{1}{2 N}\left(\dot{g}_{a b}-N^{c} g_{a b, c}-g_{c a} N_{, b}^{c}-g_{b c} N_{, a}^{c}\right) .
$$

Equations (3.6),(3.7) will be useful when we consider the reality conditions and in determining variations of $A_{0}^{i}$. Now we will continue with the canonical version of the theory.

\section{THE CANONICAL HAMILTONIAN APPROACH}

The Legendre map

$$
\mathcal{F} L: T Q \rightarrow T^{*} Q
$$

from configuration-velocity (tangent) space to phase space is defined by

$$
\mathcal{F} L(q, \dot{q})=\left(q, p=\hat{p}:=\frac{\partial L}{\partial \dot{q}}\right)
$$

we work locally, with $q, \dot{q}$ being coordinates in configuration-velocity space and $q, p$ being coordinates in phase space, as is conventional.

Our configuration variables and their conjugate canonical momenta are as follows:

$$
\begin{aligned}
& A_{\mu}^{i} \quad\left(\text { canonical momenta: } \widetilde{\pi}_{i}^{\mu}\right), \\
& \widetilde{T}_{i}^{a} \quad\left(\text { canonical momenta: } P_{a}^{i}\right), \\
& \underset{\sim}{N}(\text { canonical momentum: } \widetilde{P}), \\
& N^{a} \quad\left(\text { canonical momenta: } \widetilde{P}_{a}\right) .
\end{aligned}
$$

The primary constraints, consequences of the Lagrangian definition of the momenta, are

$$
\begin{aligned}
\widetilde{P} & =0, \\
\widetilde{P}_{a} & =0, \\
\tilde{\pi}_{i}^{\mu} & =0, \\
P_{a}^{i}-i A_{a}^{i} & =0 .
\end{aligned}
$$

The canonical Hamiltonian $H_{c}$ is defined as a function in phase space such that its pullback to tangent space under the
Legendre map is the Lagrangian energy $E_{L}$ from Eq. (2.1), that is, $E_{L}=\mathcal{F} L^{*}\left(H_{\mathrm{c}}\right) . H_{\mathrm{c}}$ is uniquely defined up to primary constraints. We take

$$
H_{c}=\int d^{3} x\left(i A_{0}^{i} \mathcal{D}_{a} \widetilde{T}_{i}^{a}-i N^{a} \widetilde{T}_{i}^{b} F_{a b}^{i}-\frac{1}{2} N \widetilde{T}_{i}^{a} \widetilde{T}_{j}^{b} F_{a b}^{i j}\right) .
$$

The constraints $P_{a}^{i}-i A_{a}^{i}=0$ and $\tilde{\pi}_{i}^{a}=0$ are second class in the sense of Dirac and can be readily disposed of; in the process, we eliminate the conjugate variables $A_{a}^{i}$ and $\tilde{\pi}_{i}^{a}$. The recipe is to put $A_{a}^{i}=-i P_{a}^{i}$ and $\tilde{\pi}_{i}^{a}=0$ everywhere in the Hamiltonian. In fact, we do not even need to substitute $-i P_{a}^{i}$ for $A_{a}^{i}$ : Since $P_{a}^{i}$ was not present in $H_{c}$, we can just take $i A_{a}^{i}$ to be the momentum variable canonically conjugate to $\widetilde{T}_{i}^{a}$. The rest of the variables are pairs of conjugate variables whose Dirac brackets coincide with the Poisson brackets.

We have achieved a canonical Hamiltonian $H_{c}$, and a number of canonical variables with Poisson brackets (actually Dirac brackets),

$$
\begin{gathered}
\left\{N_{\sim}, \widetilde{P}^{\prime}\right\}=\delta^{3}\left(x-x^{\prime}\right), \\
\left\{N^{a}, \widetilde{P}_{b}^{\prime}\right\}=\delta_{b}^{a} \delta^{3}\left(x-x^{\prime}\right), \\
\left\{\widetilde{T}_{i}^{a}, A^{\prime}{ }_{b}^{j}\right\}=-i \delta_{b}^{a} \delta_{i}^{j} \delta^{3}\left(x-x^{\prime}\right), \\
\left\{A_{0}^{i}, \widetilde{\pi}_{j}^{\prime} 0\right\}=\delta_{j}^{i} \delta^{3}\left(x-x^{\prime}\right) .
\end{gathered}
$$

The Dirac Hamiltonian, which governs the time evolution of the system, is constructed by adding to $H_{\mathrm{c}}$ the primary constraints multiplied by arbitrary functions:

$$
H_{D}=H_{c}+\int d^{3} x\left(\underset{\sim}{\lambda} \widetilde{\widetilde{P}}+\lambda^{a} \widetilde{P}_{a}+\lambda^{i} \widetilde{\pi}_{i}^{0}\right) .
$$

The second class primary constraints having been already eliminated, all the remaining primary constraints are first class.

The equations of motion derived from $H_{\mathrm{D}}$ for $\widetilde{T}_{i}^{a}$ and $A_{a}^{i}$ are

$$
\begin{aligned}
& \dot{\tilde{T}}_{i}^{a}=\epsilon^{i j k} \widetilde{T}_{k}^{a} A_{0}^{j}+2 \mathcal{D}_{b}\left(N^{[b} \widetilde{T}_{i}^{a]}\right)-i \epsilon^{i j k} \mathcal{D}_{b}\left(N \widetilde{T}_{j}^{b} \widetilde{T}_{k}^{a}\right), \\
& \dot{A}_{a}^{i}=\mathcal{D}_{a} A_{0}^{i}+N^{b} F_{b a}^{i}-i N \widetilde{T}_{j}^{b} F_{a b}^{i j} .
\end{aligned}
$$

The equations obtained from the stabilization of the primary first class constraints yield the three secondary constraints

$$
\begin{aligned}
& \widetilde{\mathcal{H}}_{0}:=-\frac{1}{2} \widetilde{T}_{i}^{a} \widetilde{T}_{j}^{b} F_{a b}^{i j}=0, \\
& \widetilde{\mathcal{H}}_{a}:=-i \widetilde{T}_{i}^{b} F_{a b}^{i}=0, \\
& \widetilde{\mathcal{H}}_{i}:=-i \mathcal{D}_{a} \widetilde{T}_{i}^{a}=0 .
\end{aligned}
$$


The canonical Hamiltonian written in terms of these constraints is

$$
H_{\mathrm{c}}=\int d^{3} x\left(-A_{0}^{i} \widetilde{\mathcal{H}}_{i}+N^{a} \widetilde{\mathcal{H}}_{a}+N \underset{\sim}{\widetilde{\mathcal{H}}_{0}}\right) .
$$

Finally, the equations for the rest of the variables, $\underset{\sim}{N}, N^{a}, A_{0}^{i}$, are

$$
\dot{\sim}=\underset{\sim}{\lambda}, \quad \dot{N}^{a}=\lambda^{a}, \quad \dot{A}_{0}^{i}=\lambda^{i} .
$$

They inform us that these variables are arbitrary-gaugevariables. The secondary constraints (4.5) are all first class (their algebra will be displayed in Sec. VII). No more constraints appear.

Let us observe that the Lagrangian equations of motion for $\widetilde{T}_{i}^{a}$ and $A_{a}^{i}$ are the same as the Hamiltonian equations of motion. The constraints (4.5) appear in configurationvelocity space as the Lagrangian equations of motion for the variables $\underset{\sim}{N}, N^{a}$, and $A_{0}^{i}$. There are no equations for the time derivatives of these variables, indicating that they are gauge variables. Also, observe that equations (3.6) have the same contents as Eqs. (4.4a) and (4.5c).

Now we are ready to apply our stabilization procedure for the reality conditions to Ashtekar's version of canonical gravity.

\section{THE REALITY CONDITIONS FOR ASHTEKAR CANONICAL GRAVITY}

\section{A. The metric reality conditions}

At the very least, the metric tensor should be real: the primary metric reality conditions are

$$
\begin{gathered}
\mathfrak{F} \underset{\sim}{N}=0, \\
\mathfrak{F} N^{a}=0, \\
\mathfrak{F} \widetilde{\widetilde{e}}^{a b}=0,
\end{gathered}
$$

where $\widetilde{\widetilde{e}}^{a b}=\widetilde{T}_{i}^{a} \widetilde{T}_{i}^{b}$. It is clear that, according to Eq. (4.3), Eqs. (5.1a) and (5.1b) fix the arbitrary functions $\underset{\sim}{\lambda}$ and $\lambda^{a}$ to be real. These equations do not have any further consequence. Requirement (5.1c) is equivalent to $\mathfrak{F} g_{a b}=0$. Notice that these reality conditions will also preserve the Lorentzian signature of the metric [presuming that $N$ and $\operatorname{det}\left(T_{i}^{a}\right)$ remain nonzero].

Before applying our method of stabilization, let us recall the last result in Sec. III: The components of the Ashtekar connect ion are auxiliary variables for the Lagrangian (3.4). Recalling the definitions $(3.7 \mathrm{~d})$, we can write a portion of the equations of motion (3.6) as

$$
A_{a}^{i}-\omega_{a}^{i}=+i T_{i}^{b} K_{b a} .
$$

Thus, if we define the quantities $M_{a b}$ as

$$
M_{a b}:=-i t_{a}^{i}\left(A_{b}^{i}-\omega_{b}^{i}\right),
$$

then this portion of the equations of motion becomes

$$
K_{a b}=M_{a b} .
$$

$K_{a b}$ is a functional of the three-metric that is real and symmetric. Thus we find here a requirement that $M_{a b}$ must be real and symmetric. The symmetry is already guaranteed by the constraint $(4.5 \mathrm{c})$. That $M_{a b}$ must be real is in fact the content of the secondary reality conditions, $\mathfrak{F}\left\{g_{a b}, H_{c}\right\}=0$, as we shall now prove.

The equations of motion for $g_{a b}$ are hidden in Eq. (5.4),

$$
\dot{g}_{a b}=\left\{g_{a b}, H_{\mathrm{D}}\right\}=\left\{g_{a b}, H_{\mathrm{c}}\right\}=2 N M_{a b}+\mathcal{L}_{\vec{N}}\left(g_{a b}\right),
$$

where $\mathcal{L}_{N}$ is the Lie derivative with respect to the vector field $N^{c} \partial_{c}$. From the first term in Eq. (5.5) we extract the secondary reality conditions

$$
\mathfrak{F} M_{a b}=0
$$

as was expected.

The last term in Eq. (5.5) is a combination of the type $\eta_{\mu}^{\nu} f_{\mu}$, as discussed in Eq. (2.9), with

$$
\begin{aligned}
\eta\left(x, x^{\prime}\right)_{a b}^{c d}= & N^{e} \delta_{, e}^{3}\left(x-x^{\prime}\right) \delta_{a}^{c} \delta_{b}^{d}+N_{, a}^{c} \delta^{3}\left(x-x^{\prime}\right) \delta_{b}^{d} \\
& +N_{, b}^{d} \delta^{3}\left(x-x^{\prime}\right) \delta_{a}^{c} .
\end{aligned}
$$

We had mentioned that the stabilization procedure simplifies when $\left\{\eta, H_{\mathrm{D}}\right\}$ vanishes; a similar simplification occurs when, as here, $\left\{\eta, H_{\mathrm{D}}\right\}$ is not zero but a harmless combination of the $\lambda^{a}$ (which are real). Thanks to this fact, and applying a similar argument to show the irrelevance of the factor $N$ before $M_{a b}$ in Eq. (5.5), we are ready to consider the tertiary reality conditions.

Since $\left\{M_{a b}, H_{\mathrm{D}}\right\}=\left\{M_{a b}, H_{\mathrm{c}}\right\}$, the tertiary reality conditions are

$$
\mathfrak{F}\left\{M_{a b}, H_{\mathrm{c}}\right\}=0 \text {. }
$$

The computation of Eq. (5.8) is a bit involved. It is useful to start by writing the canonical Hamiltonian (4.1) as a sum of three terms that clearly preserve the reality of a real triad. This way we will also gain information on the structure of the Hamiltonian; this information is useful whether we consider the metric or the triad reality conditions.

The term $N^{a} \tilde{\mathcal{H}}_{a}$ [we have used the definition (4.5b)] in $H_{\mathrm{c}}$ produces a time evolution of the triad that makes it acquire an imaginary part. This part can be eliminated by a rotation generated by $\widetilde{\mathcal{H}}_{i}$. This way we obtain a unique linear combination of $\widetilde{\mathcal{H}}_{a}$ and $\widetilde{\mathcal{H}}_{i}$ that preserves the reality of a real triad. We are led to define

$$
\widetilde{\mathcal{G}}_{a}:=\widetilde{\mathcal{H}}_{a}-A_{a}^{i} \widetilde{\mathcal{H}}_{i}
$$

Then $H_{\mathrm{c}}$ is written as

$$
H_{\mathrm{c}}=\int d^{3} x\left[-\left(A_{0}^{i}-N^{a} A_{a}^{i}\right) \widetilde{\mathcal{H}}_{i}+N^{a} \widetilde{\mathcal{G}}_{a}+N \widetilde{\sim}_{0}\right] .
$$


The rotations generated by the first term in Eq. (5.9), the integrand of which is equal to $-N A_{\mu}^{i} n^{\mu} \widetilde{\mathcal{H}}_{i}$, are not real in general. But note that according to the equations of motion (3.6)

$$
N A_{\mu}^{i} n^{\mu}-i T_{i}^{b} N_{, b}=N \Omega_{\mu}^{i} n^{\mu},
$$

where we have used definitions (3.7d) and (3.7e). Since $\Omega_{\mu}^{i}$ will be real if the triad reality conditions hold, it is useful to rewrite $H_{\mathrm{c}}$ as

$$
\begin{aligned}
H_{\mathrm{c}}= & \int d^{3} x\left[-\left(A_{0}^{i}-N^{a} A_{a}^{i}-i \widetilde{T}_{i}^{b} \mathcal{D}_{b} N\right) \widetilde{\mathcal{H}}_{i}+\left(N^{a} \widetilde{\mathcal{G}}_{a}\right)\right. \\
& \left.+\left(N \widetilde{\mathcal{H}}_{0}-i \widetilde{T}_{i}^{b}\left(\mathcal{D}_{b} N\right) \widetilde{\mathcal{H}}_{i}\right)\right] .
\end{aligned}
$$

Let us display the action of these three terms of $H_{\mathrm{c}}$ on $t_{a}^{i}$ and $A_{a}^{i}$ [since we are computing Eq. (5.7), recall that $M_{a b}$ $\left.:=-i t_{a}^{i}\left(A_{b}^{i}-\omega_{b}^{i}\right)\right]$.

The first term in Eq. (5.11) is of the type

$$
\int d^{3} x B^{i} \widetilde{\mathcal{H}}_{i},
$$

with $B^{i}$ complex. It generates $\mathrm{SO}(3, C)$ rotations $(R)$ of the triad vectors, $\delta \tau$ being an infinitesimal parameter,

$$
\delta_{R}[B \delta \tau] t_{a}^{i}=-\epsilon^{i j k} B^{j} t_{a}^{k} \delta \tau,
$$

and for the connection components,

$$
\delta_{R}[B \delta \tau] A_{a}^{i}=-\mathcal{D}_{a} B^{i} \delta \tau,
$$

that is, the Yang-Mills-like gauge transformation. The variations of the Ricci rotation coefficients are computed from the variations of the triad vectors, the results being

$$
\delta_{R}[B \delta \tau] \omega_{a}^{i}=-D_{a} B^{i} \delta \tau
$$

where $D_{a}$ stands for the covariant derivative associated with the spin connection $\omega_{a}^{i}$.

The second term in Eq. (5.11) is

$$
\int d^{3} x N^{a} \widetilde{\mathcal{G}}_{a}
$$

It generates standard spatial (three-space) diffeomorphisms $(D)$, that is,

$$
\begin{aligned}
\delta_{D}[\vec{N} \delta \tau] t_{a}^{i} & =\left(N^{b} t_{a, b}^{i}+t_{b}^{i} N_{, a}^{b}\right) \delta \tau, \\
\delta_{D}[\vec{N} \delta \tau] A_{a}^{i} & =\left(N^{b} A_{a, b}^{i}+A_{b}^{i} N_{, a}^{b}\right) \delta \tau .
\end{aligned}
$$

The third term in Eq. (5.11) generates a perpendicular diffeomorphism (that is, perpendicular to the constant-time hypersurfaces) plus a gauge rotation with descriptor

$$
t \underset{\sim}{N} A_{\mu}^{i} n^{\mu}-i \widetilde{T}^{b i} \mathcal{D}_{b} N,
$$

as we will show in Sec. VI. Thus in the real triad sector it does generate real variations. These variations (which we call $\delta_{S^{\prime}}$ to distinguish them from the variations $\delta_{S}$ generated by $\widetilde{\mathcal{H}}^{0}$ ) are in fact identical to the variations generated by the scalar generator in the real triad formalism [3], although here we apply them even if the triad is not real. The resulting variation is

$$
\delta_{S^{\prime}}[N \delta \tau] \widetilde{T}_{i}^{a}=-i \epsilon^{i j k} \mathcal{D}_{b}\left(\widetilde{T}_{j}^{b} \widetilde{T}_{k}^{a}\right) \underset{\sim}{N} \delta \tau .
$$

The corresponding variation of $t_{a}^{i}$ is

$$
\delta_{S^{\prime}}[\underset{\sim}{N} \delta \tau] t_{a}^{i}=t N_{\sim} M_{a}^{b} t_{b}^{i} \delta \tau,
$$

where $M_{a}^{b}=e^{b c} M_{c a}$, with

$$
e^{a c} g_{c b}=\delta_{b}^{a} .
$$

When operating on $A_{a}^{i}$ this transformation is, on the constraint hypersurfaces,

$$
\delta_{S^{\prime}}[N \underset{\sim}{N} \delta \tau] A_{a}^{i}=-i\left[\underset{\sim}{N} \widetilde{T}_{j}^{b} F_{a b}^{i j}-\mathcal{D}_{a}\left(\widetilde{T}_{i}^{b} \mathcal{D}_{b} N\right)\right] \delta \tau .
$$

The variations of the Ricci rotation coefficients are computed from the variations of the triad vectors,

$$
\delta_{S^{\prime}}[N \delta \tau] \omega_{a}^{i}=\epsilon^{i j k} \widetilde{T}_{k}^{b} T_{j}^{c} \mathcal{D}_{b}\left(\underset{\sim}{N} M_{a c}\right) \delta \tau .
$$

Now we can compute $\left\{M_{a b}, H_{\mathrm{c}}\right\}$. The result is

$$
\begin{aligned}
\left\{M_{a b}, H_{\mathrm{c}}\right\}= & N\left(-{ }^{3} R_{a b}-M_{c}^{c} M_{a b}+2 M_{a}^{c} M_{c b}\right) \\
& +\mathcal{L}_{\vec{N}} M_{a b}+\mathcal{D}_{a} \mathcal{D}_{b} N,
\end{aligned}
$$

where the symmetry of $M_{a b}$ [guaranteed by the constraint (4.5c)] has been used, and ${ }^{3} R_{a b}$ is the three-dimensional Ricci tensor.

Therefore the tertiary reality conditions (5.7) are automatically satisfied, for all terms on the right side of Eq. (5.16) are real by way of the primary and secondary reality conditions.

Also, we have more information: The term $-{ }^{3} R_{a b}$ on the right side of Eq. (5.16) is a real functional of $g_{a b}$. Then, an immediate generalization of Eq. (2.9) shows that this term will not give further consequences in subsequent levels of stabilization. The same is true for all the other terms, though they are not exactly of the type (2.9). For instance, consider the term $N_{, a b}$ in the last term of Eq. (5.16). In stabilizing this term, notice that $\left\{N_{a b}, H_{\mathrm{D}}\right\}=\lambda_{, a b}$ which is already real. The next step $\left\{\lambda_{a b}, H_{\mathrm{D}}\right\}$ gives exactly zero.

Summing up, from the form of the right side of Eq. (5.17) we conclude that the metric reality conditions have been fully satisfied. The algorithmic procedure devised in the previous section has terminated.

\section{B. The triad reality conditions}

The primary triad reality conditions are

$$
\begin{aligned}
\mathfrak{F} \underset{\sim}{N}=0, \\
\mathfrak{F} N^{a}=0,
\end{aligned}
$$




$$
\mathfrak{F} \widetilde{T}_{i}^{a}=0 .
$$

As before, Eqs. (5.1a) and (5.1b) fix the arbitrary functions $\underset{\sim}{\lambda}$ and $\lambda^{a}$ to be real. They do not have any further consequence.

The secondary reality conditions are $\mathfrak{F}\left\{\widetilde{T}_{i}^{a}, H_{\mathrm{c}}\right\}=0$ :

$$
\begin{aligned}
\mathfrak{F}\left\{\widetilde{T}_{i}^{a}, H_{\mathrm{c}}\right\}= & \epsilon^{i j k} \widetilde{T}_{k}^{a} \mathfrak{F} A_{0}^{j}+2 \epsilon^{i j k} N^{[b} \widetilde{T}_{j}^{a]} \mathfrak{F} A_{b}^{k}-\epsilon^{b a c} N_{, b} t_{c}^{i} \\
& -\epsilon^{b a c} \epsilon^{i j k} N t_{c}^{j}\left(\Re A_{b}^{k}-\omega_{b}^{k}\right) .
\end{aligned}
$$

Using the primary triad reality condition $(5.17 \mathrm{c})$, we can write

$$
\mathfrak{R} A_{b}^{k}-\omega_{b}^{k}=-T_{k}^{d} \mathfrak{F} M_{b d} .
$$

Computing $T_{i}^{b} \mathfrak{F}\left\{\widetilde{T}_{i}^{a}, H_{\mathrm{c}}\right\}+(a \leftrightarrow b)$, we get

$$
\mathfrak{F} M_{a b}=0,
$$

where the constraint $(4.5 \mathrm{c})$ has been used. These secondary reality conditions (5.19) were expected from the calculations of the metric reality condition case. The remaining terms of Eq. (5.18) give the rest of the secondary triad reality conditions:

$$
\mathfrak{F}\left(A_{0}^{i}-N^{a} A_{a}^{i}-i \widetilde{T}_{i}^{b} \mathcal{D}_{b} N\right)=0 .
$$

Notice that the object in Eq. (5.20) which is required to be real is the coefficient of $\widetilde{\mathcal{H}}_{i}$ in $H_{\mathrm{c}}$ in Eq. (5.11).

We need not worry about the stabilization of Eq. (5.19) because this issue has been already addressed in the study of the metric reality conditions. We do have to be concerned with the stabilization of Eq. (5.20). The tertiary triad reality conditions read

$$
\mathfrak{F}\left\{\left(A_{0}^{i}-N^{a} A_{a}^{i}-i \widetilde{T}_{i}^{b} \mathcal{D}_{b} N\right), H_{\mathrm{D}}\right\}=0 .
$$

They determine the imaginary part of $\lambda^{i}$ in Eq. (4.3),

$$
\lambda^{i}=\lambda_{0}^{i}+\lambda^{a} A_{a}^{i}+i \widetilde{T}_{i}^{a} \mathcal{D}_{a} N \lambda+N^{a}\left\{A_{a}^{i}, H_{\mathrm{c}}\right\}+i\left\{\widetilde{T}^{a i}, H_{\mathrm{c}}\right\} \mathcal{D}_{a} N,
$$

where $\lambda_{0}^{i}$ is a real arbitrary function. Notice that we have reduced the gauge freedom of rotations of the triad vectors from $\mathrm{SO}(3, C)$ to $\mathrm{SO}(3, R)$.

With this determination, the Dirac Hamiltonian becomes

$$
\begin{aligned}
H_{\mathrm{D}}^{\prime}= & H_{\mathrm{c}}+\int d^{3} x\left(\lambda^{a} A_{a}^{i}+i \widetilde{T}_{i}^{a} \mathcal{D}_{a} \lambda+N_{\sim}^{a}\left\{A_{a}^{i}, H_{\mathrm{c}}\right\}\right. \\
& \left.+i\left\{\widetilde{T}_{i}^{a}, H_{\mathrm{c}}\right\} \mathcal{D}_{a} N \widetilde{\pi}_{i}^{0}+\lambda_{\sim} \widetilde{\widetilde{P}}+\lambda^{a} \widetilde{P}_{a}+\lambda_{0}^{i} \widetilde{\pi}_{i}^{0}\right),
\end{aligned}
$$

with $\underset{\sim}{\lambda}, \lambda^{a}$, and $\lambda_{0}^{i}$ all real arbitrary functions.

$H_{\mathrm{D}}^{\prime}$ is now used for time evolution. The next reality condition is

$$
\mathfrak{F}\left\{\left\{\left(A_{0}^{i}-N^{a} A_{a}^{i}-i T_{i}^{b} N_{, b}\right), H_{\mathrm{D}}^{\prime}\right\}, H_{\mathrm{D}}^{\prime}\right\}=0,
$$

which is trivially satisfied: Since now

$$
\left\{\left(A_{0}^{i}-N^{a} A_{a}^{i}-i T_{i}^{b} N_{, b}\right), H_{\mathrm{D}}^{\prime}\right\}=\lambda_{0}^{i},
$$

we have the stronger result

$$
\left\{\left\{\left(A_{0}^{i}-N^{a} A_{a}^{i}-i T_{i}^{b} N_{, b}\right), H_{\mathrm{D}}^{\prime}\right\}, H_{\mathrm{D}}^{\prime}\right\}=0,
$$

which guarantees that no further reality conditions will arise.

\section{PROJECTABILITY OF GAUGE SYMMETRIES}

In this section we will realize the full gauge group in phase space, including transformations based on spacetime diffeomorphisms and triad rotations. Two tasks are involved in this goal. The first one is to make the infinitesimal gauge transformations in configuration-velocity space projectable to phase space. From our previous experience with conventional general relativity [1], Einstein-Yang-Mills theory [2], and real triad theory [3], we know that the arbitrary functions in the infinitesimal spacetime diffeomorphisms must depend in an explicit way on the lapse and shift functions. This was sufficient in the case of general relativity, but in the latter two cases a second step was required: We needed to add a gauge rotation. We expect something similar to occur with the Ashtekar formulation.

The second task is to construct the generators of the gauge group in phase space and to check that the transformations they generate do indeed coincide with the projectable transformations in configuration-velocity space. Notice that now there is a consistency condition to be met which was not needed in our previous work: We must require that the gauge group preserve the reality conditions.

We have already calculated [3] the projectable variations of the configuration variables $\underset{\sim}{N}$ and $N^{a}$ under diffeomorphisms with

$$
x^{\mu} \rightarrow x^{\mu}-\delta_{a}^{\mu} \xi^{a}-n^{\mu} \xi^{0},
$$

where the $\xi^{\mu}$ are arbitrary functions. As in all the theories considered previously, this dependence on the lapse and shift functions is required in order to make the variations of $\underset{\sim}{N}$ and $N^{a}$ projectable under the Legendre map. The resulting variations under perpendicular diffeomorphisms $(P D)$, with descriptor $\xi^{0}$ (with $\xi^{0}=t^{-1} \xi^{0}$, which will be useful later), are

$$
\begin{gathered}
\delta_{P D}\left[\xi^{0}\right] N=\dot{\xi}^{0}+\xi^{0} N_{, a}^{a}-N^{a} \xi_{, a}^{0}, \\
\delta_{P D}\left[\xi^{0}\right] N^{a}=-N e^{a b} \xi_{, b}^{0}+N_{, b} e^{a b} \xi^{0} .
\end{gathered}
$$

The resulting variation of $\widetilde{T}_{i}^{a}$ is [3]

$$
\delta_{P D}\left[\xi^{0}\right] \widetilde{T}_{i}^{a}=-\xi^{0} \epsilon^{i j k} \Omega_{\mu}^{k} n^{\mu} \widetilde{T}_{j}^{a}-\xi^{0} \widetilde{T}_{i}^{b} \widetilde{T}_{j}^{a} K_{b}^{j}+\xi^{0} \widetilde{T}_{i}^{a} \widetilde{T}_{j}^{b} K_{b}^{j} .
$$

We can rewrite the variation of $\widetilde{T}_{i}^{a}$ in terms of the canonical variables, using the equation of motion (5.10) so that

$$
\Omega_{\mu}^{i} n^{\mu}=A_{\mu}^{i} n^{\mu}-i N^{-1} T^{a i} N_{, a} .
$$

Also, using equation of motion (3.6), we find 


$$
\begin{aligned}
-\xi^{0} \widetilde{T}_{i}^{b} \widetilde{T}_{j}^{a} K_{b}^{j}+\xi_{\sim}^{0} \widetilde{T}_{i}^{a} \widetilde{T}_{j}^{b} K_{b}^{j}= & -i \mathcal{D}_{b}\left(\epsilon^{i j k} \widetilde{T}_{j}^{b} \widetilde{T}_{k}^{a} \xi^{0}\right) \\
& +i \epsilon^{i j k} \widetilde{T}_{j}^{b} \widetilde{T}_{k}^{a} \mathcal{D}_{b} \xi^{0}
\end{aligned}
$$

The result is that

$$
\begin{aligned}
\delta_{P D}\left[\xi^{0}\right] \widetilde{T}_{i}^{a}= & \xi^{0} \epsilon^{i j k} A_{\mu}^{j} n^{\mu} \widetilde{T}_{k}^{a}-i \epsilon^{i j k} N^{-1} \widetilde{T}^{b j} \widetilde{T}_{k}^{a} \xi^{0} \mathcal{D}_{b} N \\
& -i \mathcal{D}_{b}\left(\epsilon^{i j k} \widetilde{T}_{j}^{b} \widetilde{T}_{k}^{a} \xi^{0}\right)+i \epsilon^{i j k} \widetilde{T}_{j}^{b} \widetilde{T}_{k}^{a} \mathcal{D}_{b} \xi_{\tilde{\sim}}^{0}
\end{aligned}
$$

The variation of the Ashtekar connection requires a little more work. Since under perpendicular diffeomorphisms we will be concerned only with on-shell variations (that is, variations of solutions), our task is to find the appropriate variations of the four-dimensional Ricci rotation coefficients. We begin with the three-dimensional coefficients $\omega_{a}^{i}$, which are constructed from the triad and whose variation therefore requires only Eq. (6.2). We showed in [3] that generally

$$
\begin{aligned}
\delta \omega_{a}^{i j}= & {\underset{\sim}{a} a c} \widetilde{T}^{b[i} D_{b} \delta \widetilde{T}^{j] c}+\widetilde{T}^{b[i}{ }_{\sim}^{j}{ }_{c}^{j]} t_{a}^{k} D_{b} \delta \widetilde{T}_{k}^{c} \\
& +{\underset{\sim}{t}}_{b}^{[i} D_{a} \delta \widetilde{T}^{j] b}+\sim_{c}^{k} t_{a}^{k}{ }^{[i} \widetilde{T}^{j] b} D_{b} \delta \widetilde{T}_{k}^{c} .
\end{aligned}
$$

Using Eq. (6.2) we find

$$
\begin{aligned}
\delta_{P D}\left[\xi^{0}\right] \omega_{a}^{i}= & 2 \epsilon^{i j k} T_{j}^{b} D_{[a} K_{b]}^{k} \xi^{0}+D_{a}\left(\xi^{0} n^{\mu} \Omega_{\mu}^{i}\right) \\
& -\epsilon^{i j k} T_{j}^{b} \xi_{, b}^{0} K_{a}^{k} .
\end{aligned}
$$

Note that Eq. (5.15) demonstrates that

$$
\delta_{S^{\prime}}\left[\xi^{0}\right]=\delta_{P D}\left[t \xi^{0}\right]+\delta_{R}\left[t \xi^{0} n^{\mu} \Omega_{\mu}\right] .
$$

We will calculate the variation of $\Omega_{a}^{0 i}$ in Eq. (3.7d) using the expression

$$
K_{a}^{i}:=T^{b i} K_{a b}=N T^{b i}{ }^{4} \Gamma_{a b}^{0} .
$$

The general variation of the four-dimensional Christoffel symbols ${ }^{4} \Gamma_{a b}^{0}$ under a diffeomorphism with descriptor $\epsilon^{\mu}$ is

$$
\delta^{4} \Gamma_{b c}^{0}=-{ }^{4} \Gamma_{b c}^{\sigma} \epsilon_{, \sigma}^{0}+{ }^{4} \Gamma_{\sigma c}^{0} \epsilon_{, b}^{\sigma}+{ }^{4} \Gamma_{b \sigma}^{0} \epsilon_{, c}^{\sigma}+\epsilon_{, b c}^{0}+{ }^{4} \Gamma_{b c, \sigma}^{0} \epsilon^{\sigma} .
$$

Using methods employed in [2], we find

$$
\begin{aligned}
\delta_{P D}\left[\xi^{0}\right] K_{a}^{i}= & -T_{j}^{b}\left({ }^{3} R_{a b}^{i j}+K_{a}^{i} K_{b}^{j}-K_{b}^{i} K_{a}^{j}\right) \xi^{0}+\left(T^{b i} \xi_{, b}^{0}\right)_{, a} \\
& +\epsilon^{i j k} T_{j}^{b} \xi_{, b}^{0} \omega_{a}^{k}+\epsilon^{i j k} \xi^{0} n^{\mu} \Omega_{\mu}^{j} K_{a}^{k} .
\end{aligned}
$$

Finally, substituting Eqs. (6.5) and (6.8) into

$$
\delta_{P D}\left[\xi^{0}\right] A_{a}^{i}=\delta_{P D}\left[\xi^{0}\right] \omega_{a}^{i}+i \delta_{P D}\left[\xi^{0}\right] K_{a}^{i},
$$

we find that on-shell

$$
\begin{aligned}
\delta_{P D}\left[\xi^{0}\right] A_{a}^{i}= & -i T_{j}^{b} F_{a b}^{i j} \xi^{0}-\delta_{R}\left[\xi^{0} n^{\mu} A_{\mu}-i \xi^{0} N^{-1} T^{b} N_{, b}\right] A_{a}^{i} \\
& +\delta_{R}\left[-i T^{b} \xi_{, b}^{0}\right] A_{a}^{i} .
\end{aligned}
$$

We turn finally to the variation of $A_{0}^{i}$. Results obtained in [3] are

$$
\begin{aligned}
\delta_{P D}\left[\xi^{0}\right] \Omega_{0}^{i j}= & -4 \xi^{0} N^{a} D_{[a} K_{b]}^{[i} T^{j] b}+2 N^{b} \xi_{, a}^{0} K_{b}^{[i} T^{j] a} \\
& +2 N_{, b} \xi_{, a}^{0} T^{b[i} T^{j] a}+\left(\Omega_{\mu}^{i j} n^{\mu} \xi^{0}\right)_{, 0} \\
& +2 \xi^{0} n^{\mu} \Omega_{\mu}^{[i} \Omega_{0}^{j]}
\end{aligned}
$$

and

$$
\begin{aligned}
\delta_{D}[\vec{\xi}] \Omega_{0}^{i}= & -\epsilon^{i j k} \xi^{a}\left(K_{a}^{j} T^{b k} N_{, b}+2 N T^{b j} D_{[a} K_{b]}^{k}\right) \\
& -{ }^{3} R_{b a}^{i} N^{b} \xi^{a}+\left(\xi^{a} \omega_{a}^{i}\right)_{, 0}+\epsilon^{i j k} \xi^{a} \omega_{a}^{j} \Omega_{0}^{k} .
\end{aligned}
$$

The most efficient calculation of the on-shell variation of $\Omega_{0}^{0 i}$ is accomplished by proceeding from expression (3.7e), using variations (6.1) and (6.8). For this purpose we also require the variation

$$
\delta_{P D}\left[\xi^{0}\right] N_{, a}=-\xi_{, b}^{0} N_{, a}^{b}-\xi_{, a b}^{0} N^{b}-\xi_{, 0}^{0} N^{-1} N_{, a}+\xi_{, 0 a}^{0} .
$$

The result is

$$
\begin{aligned}
\delta_{P D}\left[\xi^{0}\right] \Omega_{0}^{0 i}= & -N^{a} T_{j}^{b} \xi^{0}\left({ }^{3} R_{a b}^{i j}+K_{a}^{i} K_{b}^{j}-K_{b}^{i} K_{a}^{j}\right) \\
& +\xi_{, a}^{0}\left(2 D_{b} N^{[b} T^{a] i}-N T_{i}^{a} T_{j}^{b} K_{b}^{j}+N T_{i}^{b} T_{j}^{a} K_{b}^{j}\right) \\
& +\widetilde{T}^{b i}\left(D_{b} \xi_{\sim}^{0}\right)_{, 0}+\epsilon^{i j k} \xi^{0} n^{\mu} \Omega_{\mu}^{j} \Omega_{0}^{0 k} .
\end{aligned}
$$

Using Eqs. (6.10) and (6.13), we deduce that on-shell

$$
\begin{aligned}
\delta_{P D}\left[\xi^{0}\right] A_{0}^{i} & =-i N^{a} T_{j}^{b} F_{a b}^{i j} \xi^{0}+i\left(T^{b i} \xi_{, b}^{0}\right)_{, 0}+i \epsilon^{i j k} T^{b j} \xi_{, b}^{0} A_{0}^{k}+\left(\xi^{0} n^{\mu} A_{\mu}^{i}-i \xi^{0} N^{-1} T^{b i} N_{, b}\right)_{, 0}+\epsilon^{i j k}\left(\xi^{0} n^{\mu} A_{\mu}^{j}-i \xi^{0} N^{-1} T^{b j} N_{, b}\right) A_{0}^{k} \\
& =-i N^{a} T_{j}^{b} F_{a b}^{i j} \xi^{0}-\delta_{R}\left[\xi^{0} n^{\mu} A_{\mu}-i \xi^{0} N^{-1} T^{b} N_{, b}\right] A_{0}^{i}+\delta_{R}\left[-i T^{b} \xi_{, b}^{0}\right] A_{0}^{i} .
\end{aligned}
$$

Notice that this variation is not projectable under the Legendre map due to the presence of time derivatives of the gauge functions $A_{0}^{i}, N$, and $N^{a}$ in the next to last line of Eq. (6.14). But fortunately, the final two lines of Eq. (6.14) are a variation under a gauge rotation with the descriptor

$$
\theta^{i}=-\xi^{0} n^{\mu} A_{\mu}^{i}+i \xi^{0} N^{-1} T^{b i} N_{, b} .
$$


That means we must accompany perpendicular diffeomorphisms with a gauge rotation with the descriptor $-\theta^{i}$ to obtain a gauge variation which is projectable under the Legendre map. It is significant that on-shell, according to Eq. (5.11), $-\theta^{i}=\xi^{0} n^{\mu} \Omega_{\mu}^{i}$, so that in the real triad sector the required gauge rotation is real, and in fact we recover the same projectability condition as in the real triad formulation of general relativity [3].

Finally, we write down the variation of $A_{0}^{i}$ under a spatial diffeomorphism. Since $\Omega_{\mu}^{i}$ and $\Omega_{\mu}^{0 i}$ each transform as a fourvector under these transformations, the result is the usual Lie derivative,

$$
\delta_{D}[\vec{\xi}] A_{0}^{i}=\dot{\xi}^{a} A_{a}^{i}+\xi^{a} A_{0, a}^{i} .
$$

\section{SYMMETRY GENERATORS}

We now turn to the gauge group itself and the structure and algebra of the generators of this group.

\section{A. Group algebra}

First, we will find the transformations of nongauge variables generated by each of the secondary constraints. For this purpose let us define

$$
\begin{gathered}
R[\xi]:=\int d^{3} x \xi^{i} \widetilde{\mathcal{H}}_{i}, \\
V[\vec{\xi}]:=\int d^{3} x \xi^{a} \widetilde{\mathcal{H}}_{a}, \\
S\left[\xi^{0}\right]:=\int d^{3} x \xi^{0} \widetilde{\mathcal{H}}_{0} .
\end{gathered}
$$

These generators are written at a given time (that is not explicitly given in the notation). All brackets associated with them are equal-time brackets. These generate gauge rotations, spatial diffeomorphisms plus associated gauge rotations, and perpendicular diffeomorphisms plus associated gauge rotations, respectively. We have, for example,

$$
\begin{aligned}
\left\{\widetilde{T}_{i}^{a}, R[\xi]\right\}= & -\epsilon_{i j k} \xi^{j} \widetilde{T}_{k}^{a}:=\delta_{R}[\xi] \widetilde{T}_{i}^{a}, \\
\left\{\widetilde{T}_{i}^{a}, V[\vec{\xi}]\right\}= & \xi_{, b}^{b} \widetilde{T}_{i}^{a}+\xi^{b} \widetilde{T}_{i, b}^{a}-\xi_{, b}^{a} \widetilde{T}_{i}^{b}-\xi^{b} \epsilon_{i j k} A_{b}^{j} \widetilde{T}_{k}^{a} \\
= & \mathcal{L}_{\dot{\xi}^{\prime}} \widetilde{T}_{i}^{a}+\delta_{R}\left[\xi^{b} A_{b}\right] \widetilde{T}_{i}^{a}, \\
\left\{\widetilde{T}_{i}^{a}, S\left[\xi^{0}\right]\right\}= & -i \mathcal{D}_{b}\left(\epsilon^{i j k} \widetilde{T}_{j}^{b} \widetilde{T}_{k}^{a} \xi^{0}\right) \\
= & \delta_{P D}\left[t \xi_{\tilde{\xi}}^{0}\right] \widetilde{T}_{i}^{a}+\delta_{R}\left[\xi^{0} A_{\mu} n^{\mu}-i N^{-1} T^{b} N_{, b} \xi^{0}\right] \widetilde{T}_{i}^{a} \\
& -\delta_{R}\left[-i \widetilde{T}^{b} \mathcal{D}_{b} \xi^{0}\right] \widetilde{T}_{i}^{a} .
\end{aligned}
$$

Thus, according to our discussion following Eq. (6.15), $S\left[\xi^{0}\right]$ does indeed generate a projected variation. Notice also that we obtain a real projected variation of a real triad if we undo the imaginary rotation of the triad due to the imaginary descriptor $i \widetilde{T}_{j}^{b} \mathcal{D}_{b} \xi^{0}$ in Eq. (7.2c). The generator on nongauge variables is

$$
S^{\prime}\left[\xi^{0}\right]:=\int d^{3} x\left(\xi^{0} \widetilde{\mathcal{H}}_{0}-i\left(\mathcal{D}_{a} \xi^{0}\right) \widetilde{T}^{a i} \widetilde{\mathcal{H}}_{i}\right) .
$$

As we noted in the discussion preceding Eq. (5.14), in the real triad sector this object generates the same variations as the scalar generator $S\left[\xi^{o}\right]$ in the real triad theory [3].

It is convenient from a geometrical perspective to define generators of nongauge variables which effect pure spatial diffeomorphisms. Using Eq. (7.2b) we deduce that the required generator is

$$
D[\vec{\xi}]:=\int d^{3} x \xi^{a}\left(\widetilde{\mathcal{H}}_{a}-A_{a}^{i} \widetilde{\mathcal{H}}_{i}\right)=\int d^{3} x \xi^{a} \widetilde{\mathcal{G}}_{a} .
$$

This is the real triad sector term we isolated in Eq. (5.11).

We are now in position to calculate the entire group algebra from the transformation properties in configurationvelocity space, projected to phase space. The projections under the Legendre map of the variations of the generators are Poisson brackets of generators. The calculations parallel those in $[2,3]$, except here it is technically simpler, and conceptually rewarding, also to calculate the Poisson brackets $\left\{S\left[\xi^{0}\right], S\left[\eta^{0}\right]\right\}$ in this manner. The nonvanishing Poisson brackets are

$$
\begin{aligned}
& \{R[\xi], R[\eta]\}=-R[[\xi, \eta]], \\
& \{R[\xi], D[\vec{\eta}]\}=-R\left[\mathcal{L}_{\eta} \xi\right], \\
& \{D[\vec{\xi}], D[\vec{\eta}]\}=-D\left[\mathcal{L}_{\vec{\eta}} \vec{\xi}\right]=D[[\vec{\xi}, \vec{\eta}]] \\
& \left\{S\left[\xi^{0}\right], D[\vec{\eta}]\right\}=-S\left[\mathcal{L}_{\eta} \xi^{0}\right], \\
& \left\{S\left[\stackrel{\xi}{\xi}^{0}\right], S\left[{\underset{\sim}{\eta}}^{0}\right]\right\}=V[\vec{\zeta}],
\end{aligned}
$$

where in Eq. (7.5e)

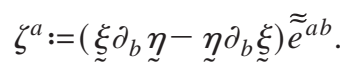

It will be useful in constructing the final complete gauge generators to have the algebra of the set $R, V$, and $S$. Using the brackets above the remaining nonvanishing brackets are

$$
\begin{gathered}
\{V[\vec{\xi}], V[\vec{\eta}]\}=V[[\vec{\xi}, \vec{\eta}]]-R\left[\xi^{a} \eta^{b} F_{a b}\right], \\
\left\{S\left[\xi^{0}\right], V[\vec{\eta}]\right\}=-S\left[\mathcal{L}_{\vec{\eta}} \xi^{0}\right]-R\left[-i \widetilde{T}_{j}^{b} F_{a b}^{i j} \eta^{a} \xi^{0}\right],
\end{gathered}
$$

where for clarity we use the notation $R\left[\xi^{i}\right]$ in the last equation instead of $R[\xi]$ as in Eq. (7.1a).

\section{B. Complete symmetry generators}

The canonical Hamiltonian in terms of the generators takes the form 


$$
H_{\mathrm{c}}=\int d^{3} x N^{A} \mathcal{H}_{A}=: N^{A} \mathcal{H}_{A},
$$

where we define

$$
N^{A}:=\left\{\underset{\sim}{N}, N^{a},-A_{0}^{i}\right\}, \quad \mathcal{H}_{A}:=\left\{\widetilde{\mathcal{H}}_{0}, \tilde{\mathcal{H}}_{a}, \widetilde{\mathcal{H}}_{i}\right\}
$$

and where spatial integrations over corresponding repeated capital indices are assumed. It was shown in [1] that the complete symmetry generators then take the form

$$
G(t)=\xi^{A} G_{A}^{(0)}+\dot{\xi}^{A} G_{A}^{(1)}
$$

the descriptors $\xi^{A}$ are arbitrary functions:

$$
\xi^{A}=\left\{{\underset{\sim}{\xi}}^{0}, \xi^{a}, \xi^{i}\right\}
$$

The simplest choice for the $G_{A}^{(1)}$ are the primary constraints $P_{A}$,

$$
P_{A}:=\left\{\widetilde{\widetilde{P}}, \widetilde{P}_{a},-\widetilde{P}_{i}:=-\widetilde{\pi}_{i}^{0}\right\},
$$

with the result that

$$
G\left[\xi^{A}\right]=P_{A} \dot{\xi}^{A}+\left(\mathcal{H}_{A}+P_{C^{\prime \prime}} N^{B^{\prime}} \mathcal{C}_{A B^{\prime}}^{C^{\prime \prime}}\right) \xi^{A},
$$

where the structure functions are

$$
\left\{\mathcal{H}_{A}, \mathcal{H}_{B^{\prime}}\right\}=: \mathcal{C}_{A B^{\prime}}^{C^{\prime \prime}} \mathcal{H}_{C^{\prime \prime}}
$$

Using the brackets calculated in the previous section we read off the following non-vanishing structure functions:

$$
\begin{aligned}
C_{0^{\prime} 0^{\prime \prime}}^{a}= & \widetilde{\widetilde{e}}^{a b}\left[-\delta^{3}\left(x-x^{\prime}\right) \partial_{b}^{\prime \prime} \delta^{3}\left(x-x^{\prime \prime}\right)\right. \\
& \left.+\delta^{3}\left(x-x^{\prime \prime}\right) \partial_{b}^{\prime} \delta^{3}\left(x-x^{\prime}\right)\right], \\
C_{b^{\prime} c^{\prime \prime}}^{a}= & -\delta^{3}\left(x-x^{\prime}\right) \partial_{b}^{\prime \prime} \delta^{3}\left(x-x^{\prime \prime}\right) \delta_{c}^{a} \\
& +\delta^{3}\left(x-x^{\prime \prime}\right) \partial_{c}^{\prime} \delta^{3}\left(x-x^{\prime}\right) \delta_{b}^{a}, \\
C_{j^{\prime} k^{\prime \prime}}^{i}= & -\epsilon^{i j k} \delta^{3}\left(x-x^{\prime}\right) \delta^{3}\left(x-x^{\prime \prime}\right), \\
C_{0^{\prime} a^{\prime \prime}}^{0}= & \delta^{3}\left(x-x^{\prime \prime}\right) \partial_{a}^{\prime} \delta^{3}\left(x-x^{\prime}\right)-\delta^{3}\left(x-x^{\prime}\right) \partial_{a}^{\prime \prime} \delta^{3}\left(x-x^{\prime \prime}\right), \\
C_{0^{\prime} a^{\prime \prime}}^{i}= & i \widetilde{T}_{j}^{b} F_{a b}^{i j} \delta^{3}\left(x-x^{\prime}\right) \delta^{3}\left(x-x^{\prime \prime}\right), \\
C_{a^{\prime} b^{\prime \prime}}^{i}= & -F_{a b}^{i} \delta^{3}\left(x-x^{\prime}\right) \delta^{3}\left(x-x^{\prime \prime}\right) .
\end{aligned}
$$

With the use of the structure functions derived above, we obtain the following generators, denoted by $G_{R}[\xi], G_{V}[\vec{\eta}]$, and $G_{S}\left[\zeta^{0}\right]$. These generate, respectively, gauge rotations, spatial diffeomorphisms, and perpendicular diffeomorphisms (plus associated gauge rotations in the last two cases):

$$
G_{R}[\xi]:=\int d^{3} x\left[\widetilde{\mathcal{H}}_{i} \xi^{i}-\widetilde{P}_{i}\left(\dot{\xi}^{i}+\epsilon_{i j k} \xi^{j} A_{0}^{k}\right)\right],
$$

$$
\begin{aligned}
& G_{V}[\vec{\eta}]:=\int d^{3} x\left[\widetilde{\mathcal{H}}_{a} \eta^{a}+\widetilde{\widetilde{P}}\left(N_{, a} \eta^{a}-N_{\sim} \eta_{, a}^{a}\right)+\widetilde{P}_{a}\left(\dot{\eta}^{a}+N_{, b}^{a} \eta^{b}\right.\right. \\
& \left.\left.-N^{b} \eta_{, b}^{a}\right)+\widetilde{P}_{i}\left(F_{a b}^{i} \eta^{a} N^{b}+i F_{a b}^{i j} \widetilde{T}_{j}^{b} N \eta^{a}\right)\right], \\
& G_{S}\left[\zeta^{0}\right]:=\int d^{3} x\left[\widetilde{\widetilde{H}}_{0} \zeta^{0}+\widetilde{\widetilde{P}}\left(\dot{\zeta}^{0}-N^{a} \varliminf_{, a}^{0}+N_{, a}^{a} \zeta^{0}\right)\right.
\end{aligned}
$$

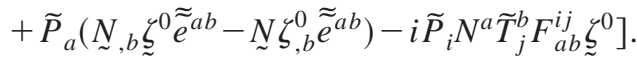

We wish to emphasize the following point: Notice that the variation of $A_{0}^{i}$ generated by $G_{S}\left[\xi^{0}\right]$ is, using Eq. (6.15),

$$
\begin{aligned}
\left\{A_{0}^{i}, G_{S}\left[\xi^{0}\right]\right\}= & -i N^{a} \widetilde{T}_{j}^{b} F_{a b}^{i j} \xi^{0} \\
= & \delta_{P D}\left[\xi^{0}\right] A_{0}^{i}+\delta_{R}\left[\xi^{0} n^{\mu} A_{\mu}\right. \\
& \left.-i \xi^{0} N^{-1} T^{b} N_{, b}\right] A_{0}^{i}-\delta_{R}\left[-i T^{b} \xi_{, b}^{0}\right] A_{0}^{i} .
\end{aligned}
$$

The second term removes the offending time derivatives of gauge variables, so that the first two variations taken together are projectable. The third variation is projectable, and in fact when combined with the variation generated by $G_{S}\left[\xi^{0}\right]$ produces a variation which conserves the reality of real triads, as we noted in defining the generator $S^{\prime}\left[\xi^{0}\right]$ in Eq. (7.3). The general relation is

$$
\begin{aligned}
\delta_{P D} & {\left[\xi^{0}\right]+\delta_{R}\left[\xi^{0} n^{\mu} A_{\mu}-i \xi^{0} N^{-1} T^{b} N_{, b}\right] } \\
= & \left\{-, G_{S}\left[\xi^{0}\right]\right\}+\left\{-, G_{R}\left[-i T^{b} \xi_{, b}^{0}\right]\right\} \\
& =:\left\{-, G_{S^{\prime}}\left[\xi_{\sim}^{0}\right]\right\} .
\end{aligned}
$$

Note that the secondary constraint term in $G_{S^{\prime}}$ is just Eq. (7.3).

Finally, we use the generators above to construct $G_{D}[\vec{\xi}]$, the complete generator of spatial diffeomorphisms with descriptor $\vec{\xi}$. Refer to Eq. (7.4); the generator is evidently, using the equation of motion $(4.4 \mathrm{~b})$,

$$
\begin{aligned}
G_{D}[\vec{\xi}]= & G_{V}[\vec{\xi}]-G_{R}\left[A_{a} \xi^{a}\right] \\
= & \int d^{3} x\left[\widetilde{\mathcal{G}}_{a} \xi^{a}+\widetilde{\widetilde{P}}\left({\underset{\sim}{N, a}}_{\xi^{a}} \xi_{\sim} \xi_{, a}^{a}\right)\right. \\
& \left.+\widetilde{P}_{a}\left(\dot{\xi}^{a}+N_{, b}^{a} \xi^{b}-N^{b} \xi_{, b}^{a}\right)+\widetilde{P}_{i}\left(\dot{\xi}^{a} A_{a}^{i}+\xi^{a} A_{0, a}^{i}\right)\right] .
\end{aligned}
$$

\section{The Hamiltonian and rigid time translation}

Now that we have the complete set of generators, we can reconstruct the Hamiltonian, recognizing that rigid (in the sense of advancing by the same infinitesimal parameter on each constant-time hypersurface) translation in time is a diffeomorphism implemented on restricted members of equivalence classes of solution trajectories. We take as given explicit spacetime functions $\xi^{0}$ and $\xi^{a}$. We restrict our 
attention to solutions for which $t \xi^{0}=N \delta \tau$ and $\xi^{a}=N^{a} \delta \tau$ for some infinitesimal parameter $\delta \tau$. However, we recall that

$$
\int d^{3} x \tilde{\xi}^{0} \widetilde{\mathcal{H}}_{0}+\int d^{3} x \xi^{a} \widetilde{\mathcal{G}}_{a}
$$

does not generate a pure diffeomorphism. We must subtract the additional gauge rotation generated by $\int d^{3} x \xi^{0} \widetilde{\mathcal{H}}_{0}$. According to Eq. (7.2c) the descriptor of this gauge rotation is

$$
\xi^{0} A_{\mu}^{i} n^{\mu}-\xi^{0} i N^{-1} T^{b i} N_{, b}+i \widetilde{T}^{b i} \mathcal{D}_{b} \xi^{0} .
$$

When we restrict this descriptor to those solutions for which $\xi^{0}=N \delta \tau$ and $\xi^{a}=N^{a} \delta \tau$, the descriptor becomes $A_{0}^{i} \delta \tau$ $-A_{a}^{i} N^{a} \delta \tau$. We deduce that the required Hamiltonian is

$$
H=\int d^{3} x N_{\sim} \widetilde{\mathcal{H}}_{0}+\int d^{3} x N^{a} \widetilde{\mathcal{H}}_{a}-\int d^{3} x A_{0}^{i} \widetilde{\mathcal{H}}_{i},
$$

where we have used the fact that $\widetilde{\mathcal{G}}_{a}=\widetilde{\mathcal{H}}_{a}-A_{a}^{i} \widetilde{\mathcal{H}}_{i}$. The Hamiltonian in Eq. (7.13) is coincident with the canonical Hamiltonian (4.6).

The gauge variables $N, N^{a}, A_{0}^{i}$ in Eq. (7.13) are now to be thought of as arbitrarily chosen but explicit functions of spacetime. This object (7.13) will then generate a time translation, which is rigid in the sense of having the same constant value $\delta \tau$ on each equal-time hypersurface, but only on those members of equivalence classes of solutions for which the dynamical variables $N, N^{a}, A_{0}^{i}$ have the same explicit functional forms. On all other solutions the corresponding variations correspond to more general diffeomorphism and gauge transformations.

In fact, as we pointed out in [2], every generator $G\left[\xi^{A}\right]$ in Eq. (7.9) with $\xi^{0}>0$ may be considered to be a Hamiltonian in the following sense:

$$
G\left[\xi^{A}\right]=G_{R}[\xi]+G_{D}[\vec{\xi}]+G_{S}\left[\xi^{0}\right]
$$

generates a global time translation on those solutions which have

$$
\begin{array}{r}
N \delta \tau=\xi^{0}, \\
N^{a} \delta \tau=\xi^{a}, \\
\left(-A_{0}^{i}+A_{a}^{i} N^{a}\right) \delta \tau=\xi^{i} .
\end{array}
$$

We have already demonstrated this fact for the nongauge variables, and it is instructive to verify the claim for the gauge variables $N, N^{a}$, and $A_{0}^{i}$. The demonstration for $N$ and $N^{a}$ is given in [2]. Substituting Eq. (7.14) into Eqs. (7.11a), (7.11c),(7.11e), we have

$$
\begin{aligned}
\delta A_{0}^{i}= & {\left[-\left(-A_{0}^{i}+A_{a}^{i} N^{a}\right)_{, 0}-\epsilon^{i j k}\left(-A_{0}^{j}+A_{a}^{j} N^{a}\right) A_{0}^{k}\right.} \\
& +A_{a}^{i} \dot{N}^{a}+N^{a} \dot{A}_{a}^{i}+i F_{a b}^{i j} T_{j}^{b} N^{a} N+\epsilon^{i j k} N^{a} A_{a}^{j} A_{0}^{k} \\
& \left.-i F_{a b}^{i j} T_{j}^{b} N^{a} N\right] \delta \tau \\
= & \dot{A}_{0}^{i} \delta \tau .
\end{aligned}
$$

\section{Finite real gauge transformations}

We close this section by noting that the arguments presented in Sec. V demonstrating the preservation of reality conditions under time evolution apply almost unaltered to finite arbitrary symmetry transformations. The only restrictions which must be placed on the descriptors $\xi^{i}$ and $\xi^{a}$ are that they be real. The triad reality condition implies in addition that we must employ the generator $G_{S^{\prime}}\left[\xi^{0}\right]$, defined in Eq. (7.11d), instead of $G_{S}\left[\xi^{0}\right]$, defined in Eq. (7.2c), and the descriptor $\xi^{0}$ must be real. Then we find, as in Eq. (5.18), with the simple substitutions $\underset{\sim}{N} \rightarrow \tilde{\xi}^{0}, N^{a} \rightarrow \xi^{a}$, that

$$
\mathfrak{F}\left\{\widetilde{T}_{i}^{a}, G_{S^{\prime}}\left[\xi_{\tilde{z}}^{0}\right]\right\}=0,
$$

when $\xi^{0}$ is real. The next and higher levels of reality stabilization are satisfied, just as in Sec. V, with the substitutions $N \rightarrow \xi^{0}, N^{a} \rightarrow \xi^{a}$.

The complete infinitesimal gauge generator which respects the triad reality condition is

$$
G_{\text {real }}\left[\xi^{A}\right]:=G_{R}[\xi]+G_{D}[\vec{\xi}]+G_{S^{\prime}}\left[\xi^{0}\right],
$$

where $\xi^{A}$ are real (if one has only the metric reality conditions, then only $\vec{\xi}$ and $\xi^{0}$ need be real). Finally, the finite real generator (which complies with the triad reality conditions), for finite parameter $\tau$, is

$$
\mathcal{T} \exp \left(\int_{t_{0}}^{t_{0}+\tau} d t\left\{-, G_{\text {real }}\left[\xi^{A}\right]\right\}\right) .
$$

\section{COUNTING THE DEGREES OF FREEDOM}

\section{A. With the metric reality conditions}

Let us again stress the relevant role of the variables in Eq. (5.3):

$$
M_{a b}:=-i t_{a}^{i}\left(A_{b}^{i}-\omega_{b}^{i}\right) .
$$

We substitute

$$
A_{a}^{i}=\omega_{a}^{i}+i T_{i}^{b} M_{b a}
$$

into the constraints (4.5a) and (4.5b) [remember that the content of Eq. (4.5c) is the condition that $M_{a b}$ be symmetric]. We get, for Eq. (4.5a) $\left({ }^{3} R\right.$ is the three-Ricci scalar),

$$
{ }^{3} R+\left(M_{a}^{a}\right)^{2}-M_{b}^{a} M_{a}^{b}=0,
$$

and for Eq. (4.5b),

$$
{ }^{3} \nabla_{a} M_{b}^{b}-{ }^{3} \nabla_{b} M_{a}^{b}=0 .
$$

These are the standard scalar and vector constraints for canonical Arnowitt-Deser-Misner (ADM) general relativity [24]. This is an expected result, because $M_{a b}$ gives, according to Eq. (5.4), the initial values for the components of the extrinsic curvature.

The initial data are, therefore: $N, N^{a}, M_{a b}$, all real with $M_{a b}$ symmetric, and $t_{a}^{i}, A_{0}^{i}$, complex. Thus we are imple- 
menting the constraints $(4.5 \mathrm{c})$ and the secondary reality condition (5.6). $A_{a}^{i}$ is then determined by Eq. (8.1). This amounts to $1+3+6+2 \times(9+3)=34$ real pieces of data. But $t_{a}^{i}$ must satisfy the six restrictions coming from the first metric reality condition (5.1), and both $M_{a b}$ and $t_{a}^{i}$ must fulfill the four constraints (8.2) and (8.3). The number of independent real pieces of data is then $34-6-4=24$.

Now let us turn to the gauge freedom. We have the four generators corresponding to the space-time diffeomorphisms and the six generators for $\mathrm{SO}(3, C)$, three for real rotations and three for imaginary rotations. This totals 10 generators. All these generators, as we have seen in the previous section, contain primary and secondary first class constraints. This means that we must spend two gauge fixing constraints for each generator-see, for example, [4] for the theory of gauge fixing. Hence we must produce $2 \times 10=20$ gauge fixing constraints to eliminate fully the unphysical degrees of freedom. The final counting of physical degrees of freedom is therefore $24-20=4$. This is the standard number of degrees of freedom of general relativity.

\section{B. With the triad reality conditions}

Now the initial data are: $N, N^{a}, M_{a b}, t_{a}^{i}$, and $\Re A_{0}^{i}$, all real with $M_{a b}$ symmetric. In this way we have already implemented the primary and secondary triad reality conditions. $A_{a}^{i}$ is determined, as before, by Eq. (8.1), and the imaginary part of $A_{0}^{i}$ is determined by Eq. (5.20). This amounts to 1 $+3+6+9+3=22$ real pieces of data. But $t_{a}^{i}$ and $M_{a b}$ are still constrained to satisfy the $4 \mathrm{ADM}$ constraints (8.2) and (8.3). The number of independent real data is then $22-4$ $=18$.

Now let us turn to gauge freedom. We have the four generators corresponding to the spacetime diffeomorphisms and the three generators that are left after reducing $\mathrm{SO}(3, C)$ to $\mathrm{SO}(3, R)$ in order to preserve Eq. (5.20). This totals seven generators. As we have mentioned above, we must introduce two gauge fixing constraints for each generator. The final counting of physical degrees of freedom is, again, 18-14 $=4$.

\section{CONCLUSIONS}

In this paper we have given a full account of two issues concerning the complex Ashtekar approach to canonical gravity: the nature of the gauge group and the implementation of reality conditions. We have solved the problem of the projectability of the spacetime diffeomorphism transformations from configuration-velocity space to phase space; we have constructed the complete set of canonical generators of the gauge group in phase space (which includes the gauge variables); and we have verified that they indeed generate the projected gauge transformations obtained from configuration-velocity space. This result proves that the canonical formalism is capable of displaying all the gauge structure of the theory, including the time diffeomorphisms, and in particular it proves that the gauge group in configuration-velocity space is the same as in phase space- the only difference is a matter of a convenient basis for the generators.

The gauge rotations which must be added to spacetime diffeomorphisms to achieve projectability differ somewhat from the Einstein-Yang-Mills case (see [2]). The difference is due to the fact that the Ashtekar connection is not a manifest spacetime one-form under diffeomorphisms for which the descriptor $\epsilon_{, a}^{0} \neq 0$.

The full projectable transformation group must be interpreted as a transformation group on the space of solutions of the equations of motion. The pullback of variations of $A_{a}^{i}$ from phase space to configuration-velocity space yields variations $\delta \dot{\widetilde{T}}_{i}^{a}$ which only coincide on-shell with $(d / d t) \widetilde{T}_{i}^{a}$. However, if we use only the pullback of the variations of the configuration variables, ignoring the pullback of momentum variables, the resulting variation of the Lagrangian is a divergence (note that we have been ignoring boundary termsfor a discussion of the algebra of spatial diffeomorphisms including boundary terms, see [25]). These pullbacks yield Noether Lagrangian symmetries. For details see $[2,26]$.

This restriction to solution trajectories is intimately related to our demonstration that all $G\left[\xi^{A}\right]$ generators (with $\xi^{0}>0$ ) can be interpreted as Hamiltonians (for time evolution, in the sense discussed in Sec. VII).

Since the complex character of the Ashtekar connection introduces the issue of reality conditions, we have first produced a general theoretical framework for the stabilization algorithm for these conditions. We showed that there are striking differences from Dirac's method of stabilization of constraints (reality conditions are not constraints in the Dirac sense). For instance, the calculation that shows that the stabilization procedure has been completed is typically not nearly as straightforward as in the Dirac case.

Our display of the reality conditions for Ashtekar's formulation is not new, but we present a rigorous proof, based on the stabilization algorithm, that the set of reality conditions and the algorithmic computation are complete. Also, in the case of the triad reality conditions, we showed that the stabilization algorithm implies the partial determination of some of the arbitrary functions (actually, the determination of their imaginary parts) in the Dirac Hamiltonian $H_{D}$. We have proved that the reality conditions are consistent with the gauge group.

We note two links between the triad reality conditions and the canonical generators associated with projectable diffeomorphisms. First, the form of our generator (7.4) for spatial diffeomorphisms of the nongauge variables is the same as the form of the generator (5.8) dictated by the triad reality conditions. In contrast, in the Einstein-Yang-Mills case [2], the form of this generator was more a matter of convenience than necessity. Second, the form of the canonical Hamiltonian in Eq. (5.11) was suggested by triad reality conditions. When $\underset{\sim}{N}$ is replaced by $\xi^{0}$ in the third term in the integrand, one obtains the generator (7.3) of the canonical version of the perpendicular diffeomorphisms - when a rotation is subtracted to make these diffeomorphisms projectable; this rotation cancels the next to last term in Eq. (6.15). In fact, the rotation which is subtracted is identified as being 
a real rotation within the triad reality conditions [see Eq. (5.20)].

Finally, we presented the counting of degrees of freedom, either under the metric reality conditions or the triad reality conditions. We showed this number matches the standard number of degrees of freedom of general relativity.

We feel that this work provides a new understanding of spacetime diffeomorphisms in the full (that is, including the gauge variables) complex canonical formalism of Ashtekar for gravity. We expect that implications for an eventual quantum theory of gravity will include insights into the prob- lem of time in such a theory. We will be investigating these ideas further [27].

\section{ACKNOWLEDGMENTS}

J.M.P. and D.C.S. would like to thank the Center for Relativity of The University of Texas at Austin for its hospitality. J.M.P. acknowledges support by CICYT, AEN980431, and CIRIT, GC 1998SGR, and wishes to thank the Comissionat per a Universitats i Recerca de la Generalitat de Catalunya for a grant. D.C.S. acknowledges support by National Science Foundation Grant PHY94-13063.
[1] J. M. Pons, D. C. Salisbury, and L. C. Shepley, Phys. Rev. D 55, 658 (1997).

[2] J. M. Pons, D. C. Salisbury, and L. C. Shepley, "Gauge Transformations in Einstein-Yang-Mills Theories,'” gr-qc/9912086.

[3] J. M. Pons, D. C. Salisbury, and L. C. Shepley, "The gauge group in the real triad formulation of general relativity," gr-qc/9912087.

[4] J. M. Pons and L. C. Shepley, Class. Quantum Grav. 12, 1771 (1995).

[5] A. Ashtekar, Phys. Rev. Lett. 57, 2244 (1986).

[6] A. Ashtekar, Phys. Rev. D 36, 1587 (1987).

[7] A. Ashtekar, Lectures on Non-Perturbative Canonical Gravity, Notes prepared in collaboration with R. S. Tate (World Scientific, Singapore, 1991), and references therein.

[8] P. A. M. Dirac, Can. J. Math. 2, 129 (1950).

[9] P. A. M. Dirac, Lectures on Quantum Mechanics (Yeshiva University Press, New York, 1964).

[10] J. F. Barbero, Phys. Rev. D 51, 5507 (1995).

[11] S. Holst, Phys. Rev. D 53, 5966 (1996).

[12] G. Immirzi, Class. Quantum Grav. 14, L177 (1997).

[13] T. Thiemann, Class. Quantum Grav. 13, 1383 (1996).

[14] A. Ashtekar, Phys. Rev. D 53, R2865 (1996).
[15] G. A. Mena Marugán, “Geometric interpretation of Thiemann's generalized Wick transform," gr-qc/9705031.

[16] P. G. Bergmann and G. J. Smith, Phys. Rev. D 43, 1157 (1991).

[17] G. Yoneda and H. Shinkai, Class. Quantum Grav. 13, 783 (1996).

[18] J. Samuel, Pramana J. Phys. 28, L429 (1987).

[19] T. Jacobson and L. Smolin, Phys. Lett. B 196, 39 (1987).

[20] T. Jacobson and L. Smolin, Class. Quantum Grav. 5, 583 (1988).

[21] D. C. Salisbury, A. Adams, D. Mann, L. Turvan, B. Turner, and L. C. Shepley, Class. Quantum Grav. 11, 2789 (1994).

[22] C. Rovelli, Class. Quantum Grav. 8, 1613 (1991).

[23] P. Peldán, Class. Quantum Grav. 11, 1087 (1994).

[24] R. Arnowitt, S. Deser, and C. W. Misner, in Gravitation: An Introduction to Current Research, edited by L. Witten (Wiley, New York, 1962), pp. 227-265.

[25] V. O. Soloviev, Theor. Math. Phys. 112, 906 (1997).

[26] X. Gràcia and J. M. Pons (in preparation).

[27] D. C. Salisbury, J. M. Pons, and L. C. Shepley, "Gauge symmetries in Ashtekar's formulation of general relativity," gr-qc/0004013. 